\title{
Global Fibrinolytic Capacity Is Decreased in Polycystic Ovary Syndrome, Suggesting a Prothrombotic State
}

\author{
BÜLENT O. YILDIZ, İBRAHIM C. HAZNEDAROǦLU, ŞERAFETTİN KİRAZLI, AND \\ MIYASE BAYRAKTAR
}

Division of Endocrinology and Metabolism (B.O.Y., M.B.) and Division of Hematology (İ.C.H., S.K.), Department of Internal Medicine, Faculty of Medicine, Hacettepe University, Ankara, Turkey, 06100

\begin{abstract}
The polycystic ovary syndrome (PCOS) is associated with an increased risk of cardiovascular disease (CVD). Insulin resistance (IR), hyperandrogenism, and dyslipidemia are wellknown cardiovascular risk factors in PCOS. Impaired fibrinolysis could also contribute to the development of CVD in PCOS. Global fibrinolytic capacity (GFC) is a recently developed method, which is reflected by the amount of generated D-dimer when the fibrinolysis of a freeze-dried fibrin clot is stopped by introducing aprotinin. GFC is sensitive to all the factors involved in the process of fibrinolysis. We evaluated whether women with PCOS have any alterations in the GFC and other essential hemostatic parameters. Fifty-nine nonobese, normal glucose-tolerant women with PCOS (age, $22.9 \pm$ $4.4 \mathrm{yr}$; body mass index, $23.0 \pm 2.4 \mathrm{~kg} / \mathrm{m}^{2}$ ) and 23 age- and body mass index-matched healthy controls participated. We measured GFC and triglycerides; total cholesterol; HDL-cholesterol (HDL-C); lipoprotein-a; prothrombin time; partial
\end{abstract}

thromboplastin time; thrombin time; antithrombin III; factors II, V, VII, and X; fibrinogen; plasminogen; antiplasmin; and D-dimer. Serum glucose and insulin (at baseline and during a 75-g 2-h oral glucose tolerance test) were also measured, and IR was assessed by homeostatic model assessment. GFC was significantly lower in the PCOS group, compared with the control group $(2.49 \pm 1.6 v s .5 .95 \pm 2.43 \mu \mathrm{g} / \mathrm{ml}, P<0.001)$. All the other coagulation and fibrinolysis parameters were comparable between the two groups. The PCOS group had lower HDL-C and higher IR values. GFC was correlated with testosterone and free testosterone negatively and with HDL-C positively. There was no correlation between GFC and any of the IR parameters. Our results suggest that women with PCOS have impaired fibrinolysis, as reflected by the decreased GFC. This impairment is not related to the IR and may increase the risk of CVD in PCOS. (J Clin Endocrinol Metab 87: 3871-3875, 2002)
$\mathrm{P}$ OLYCYSTIC OVARY SYNDROME (PCOS) is the most common endocrine disorder of reproductive-aged women, characterized by hyperandrogenism and chronic anovulation (1). The disorder affects approximately $4-8 \%$ of women of reproductive age $(2,3)$. Current literature supports the fact that women with PCOS have increased risk of cardiovascular disease (CVD) (4). However, there is no direct evidence of increased morbidity or mortality from CVD in PCOS $(5,6)$. Insulin resistance (IR), obesity, dyslipidemia, hypertension, and hyperandrogenism constitute a cluster of cardiovascular risk factors in PCOS (7-13).

Abnormalities in the coagulation and fibrinolytic pathways could also contribute to the development of CVD in PCOS. There are conflicting reports on hemostasis in PCOS. Both increased $(14,15)$ and normal $(16,17)$ levels of plasminogen activator inhibitor 1 (PAI-1) were reported. Increased (15) and decreased (16) levels of fibrinogen were determined. On the other hand, normal values of von Wil-

Abbreviations: A, Androstenedione; AP, antiplasmin; aPTT, activated partial thromboplastin time; ATIII, antithrombin III; AUC, area under the curve; $\mathrm{BMI}$, body mass index; $\mathrm{CV}$, coefficient of variation; CVD, cardiovascular disease; DHEAS, dehydroepiandrosterone sulfate; FG:I, fasting glucose-to-insulin ratio; fT, free testosterone; GFC, global fibrinolytic capacity; HDL-C, high-density lipoprotein cholesterol; HOMA, homeostatic model assessment; IR, insulin resistance; Lp-a, lipoprotein-a; OGTT, oral glucose tolerance test; PAI-1, plasminogen activator inhibitor 1; PCOS, polycystic ovary syndrome; PLG act, plasminogen activity; PT, prothrombin time; $\mathrm{T}$, total testosterone; TG, triglycerides; $\mathrm{tPA}$, tissue-type plasminogen activator; TT, thrombin time; vWF, von Willebrand factor; WHR, waist to hip ratio. lebrand factor (vWF), factor VII activity (16), protein C, protein $S$, antithrombin III (ATIII), activated protein $C$ resistance (18), plasminogen, and $\alpha 2$ antiplasmin (AP) levels (17) were documented in PCOS. Many of the proteins of coagulation and fibrinolysis are involved in the pathobiology of CVD. The suppression of fibrinolysis attributable to alterations of the proteins in the fibrinolytic system are associated with the development of myocardial infarction (19). The most important limitation of the above-mentioned studies is that they failed to search a global view of fibrinolysis and/or coagulation. All of them tried to look for a single part or a few elements of the complex puzzle of hemostasis in PCOS.

Global fibrinolytic capacity (GFC) is a recently developed assay that is reflected by the amount of generated D-dimer when the fibrinolysis of a freeze-dried fibrin clot is stopped by introducing aprotinin. GFC is sensitive to reflecting all molecular components involved in the process of fibrinolysis (20-26). The GFC assay allows exploration of the fibrinolytic potential of plasma and evaluation of fibrinolytic dysfunctions in a reliable manner. The main principle of the GFC method is that, in the presence of a constant and limited amount of exogenous tissue-type plasminogen activator (tPA), D-dimer generated from a standardized fibrin quantity is measured. For this purpose, a standardized fibrin tablet (containing silica and completely lacking plasminogen) is introduced into a $200-\mu 1$ plasma sample supplemented with a constant and limited amount of tPA. The mixture is then incubated for $1 \mathrm{~h}$ at $37 \mathrm{C}$. Fibrinolysis is thereafter stopped by introducing $50 \mu \mathrm{l}$ of an excess of apro- 
tinin. Generated D-dimer was measured by the semiquantitative macrolatex agglutination technique. The principle of the GFC assay was depicted in Fig. 1. Amiral et al. (20) noticed no difference whatever whether the assay was performed on whole blood, platelet-rich plasma, or platelet-poor plasma, or on heparin- or hirudin-anticoagulated specimen. GFC was also classified according to its efficacy and scored from $0-4$. The score of 0 corresponded to less than $1 \mu \mathrm{g} / \mathrm{ml}$ generated D-dimer concentration, whereas the score 4 referred to a level of more than $20 \mu \mathrm{g} / \mathrm{ml}$ generated D-dimer. The effects of tPA, prourinary type plasminogen activator, urinary type plasminogen activator, $\alpha 2 \mathrm{AP}$, hystidin-rich glycoproteins, PAI-1, plasminogen, activated protein C, and factor XII were evaluated in the GFC assay. Because GFC assay explores plasma fibrinolytic potential, its performance has been evaluated in a variety of clinical settings associated with fibrinolytic dysfunctions. Fibrinolysis is initiated, regulated, and acts in the microenvironment. The fibrinolytic activity is then diluted and inactivated in the circulating blood. However, there is a stable residual plasma fibrinolytic activity, which reflects the body's fibrinolytic potential. This residual fibrinolytic activity closely relates to prevention of thrombosis. The pioneer studies by Amiral et al. (23) disclosed that GFC can help to identify individuals with a fibrinolytic dysfunction. Particularly, the Thrombocheck Study, which studied GFC in a large population in 685 normal individuals (337 males and 348 females), 303 hypercholesterolemic individuals (232 males and 71 females), and 210 diabetic patients (65 noninsulin-dependent diabetes mellitus patients and 145 insulin-dependent diabetes mellitus patients) revealed that the GFC method is a practical and sensitive measure of fibrinolytic activity in health and disease (23). Studies in our laboratory also indicated that GFC is a sensitive method reflecting ongoing subclinical or overt prothrombotic/ hypofibrinolytic states, including Behcet's disease and others (24-26).

The aim of this study was to assess whether women with PCOS have any alterations in the GFC and the other essential hemostatic parameters. Elucidation of GFC in PCOS can reflect net fibrinolytic capacity of those patients who are prone to CVD. The advantage and unique approach in our study is to put forward a global view of fibrinolysis in this hypercoagulable enigmatic disease.

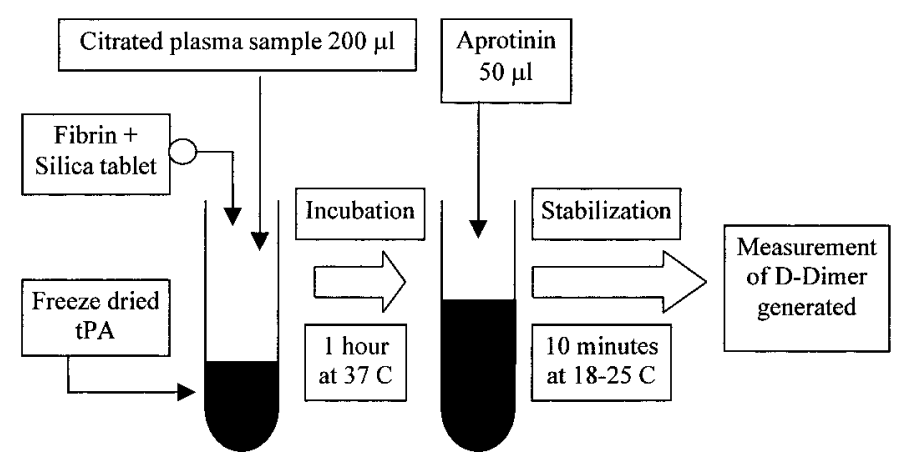

FIG. 1. Principle of the GFC assay.

\section{Subjects and Methods}

\section{Subjects}

Fifty-nine women with PCOS [mean age, $22.9 \pm 4.4 \mathrm{yr}$; mean body mass index (BMI), $\left.23.0 \pm 2.4 \mathrm{~kg} / \mathrm{m}^{2}\right]$ and 23 age- and BMI-matched healthy controls were recruited. Diagnosis of PCOS was based on the elevation of total testosterone ( $\mathrm{T}$ ) or free testosterone (fT) levels associated with chronic oligomenorrhea. Oligomenorrhea was defined as bleeding episodes occurring less than six times per year. Cushing's syndrome, nonclassical 21-hydroxylase deficiency, hyperprolactinemia, thyroid dysfunction, and androgen-secreting tumors were excluded, according to the recommendations of the National Institute of Child Health and Human Development consensus conference on PCOS (1). Subjects were not taking any medication for at least 3 months before the study. Informed consent was obtained from each subject. Waist to hip ratio (WHR) was determined (waist, midway between the lower rib margin and the iliac crest; hip, widest circumference over the great trochanters), and a 2-h 75-g oral glucose tolerance test (OGTT, glucose and insulin levels at $0,30,60,90$, and $120 \mathrm{~min}$ ) was performed. Women with impaired fasting glucose, impaired glucose tolerance, or diabetes were excluded (27).

\section{Study protocol}

All sampling procedures were performed in a fasting state, between 0800-0900 h, to avoid the effect of diurnal variation of the hemostatic system. Blood samples were drawn from large antecubital veins of the forearm, without interruption of venous flow for GFC, and other hemostasis tests [including prothrombin time (PT); activated partial thromboplastin time (aPTT); thrombin time (TT); ATIII; factors II, V, VII, and $\mathrm{X}$; fibrinogen; plasminogen activity (PLG act); $\mathrm{AP}, \mathrm{D}$-dimer; $\mathrm{T}$; $\mathrm{fT}$; androstenedione $(\mathrm{A})$; dehydroepiandrosterone sulfate (DHEAS); total cholesterol; triglycerides (TG); high-density lipoprotein cholesterol (HDL-C); and lipoprotein-a (Lp-a)].

\section{Assays}

Blood samples for GFC and PT; aPTT; TT; ATIII; factors II, V, VII, and $X$; fibrinogen; PLG act; AP; and D-dimer were centrifuged, within $30 \mathrm{~min}$ of collection, at $4 \mathrm{C}$ for $20 \mathrm{~min}$ at $3000 \mathrm{rpm}$. The supernatant plasma samples obtained were transferred into polypropylene tubes and stored, for up to 1 month, at $-30 \mathrm{C}$, until assayed.

Plasma GFC levels were measured by the semiquantitative macrolatex agglutination technique using a commercially available assay (Global Fibrinolytic Capacity STA Liatest D-Di; Diagnostica Stago, Asniëres, France) Commercially available reagents, provided by the Diagnostica Stago, were used to measure PT (STA Neoplastin Plus kit), aPTT (STA APTL LT kit), TT (STA TT kit), AT-III (STA Antithrombin III kit), F II (STA factor II kit), F V (STA factor V kit), F VII (STA factor VII kit), F X (STA factor X kit), DD (STA liatest D-Di kit), fibrinogen (STA fibrinogen kit), PLG act (STA stachrom plasminogen kit), and AP (STA stachrom AP kit). For all determinations, the manufacturers' instructions were followed. The ranges of intra- and interassay coefficients of variation (CVs) of our laboratory were $5.2-8.7 \%$ and $6.5-9.4 \%$, respectively.

Plasma glucose was measured by the glucose oxidase technique (Roche Molecular Biochemicals, Mannheim, Germany). T and DHEAS were measured by chemiluminescent enzyme immunoassay (Immulite 2000; Diagnostic Products, Los Angeles, CA), with an average interassay $\mathrm{CV}$ of $8 \%$ and intraassay CV of $7 \%$. A, fT, and insulin were measured by RIA (Diagnostic Systems Laboratories, Inc., Webster, TX). The average interassay and intraassay CV's were $6.7 \%$ and $6.4 \%$, respectively.

Plasma total cholesterol, TG, and HDL-C levels were determined by enzymatic colorimetric method (Roche Molecular Biochemicals, Mannheim, Germany). The average interassay and intraassay CV's were $2.2 \%$ and $1.4 \%$, respectively. Plasma Lp-a levels were determined by the rate nephelometry method (chemical analyzer systems; Beckman, Fullerton, CA).

Using glucose and insulin data from the OGTT, glucose and insulin areas under the curves $\left(\mathrm{AUC}_{\text {glucose }}\right.$ and $\mathrm{AUC}_{\text {insulin }}$ ) during OGTT, as well as fasting glucose-to-insulin ratio (FG:I) were calculated. Homeostatic model assessment (HOMA) (28) is applied by using the following formula: Insulin resistance $(\mathrm{HOMA} I R)=$ fasting insulin $(\mu \mathrm{U} / \mathrm{ml}) \times$ fasting glucose $(\mathrm{mm}) \div 22.5$. 


\section{Statistical analysis}

Data analysis was performed by using the 9 PC Package (SPSS, Inc., Chicago, IL). All parameters were given as mean $\pm \mathrm{sD}$. Student's $t$ test was used to analyze differences between the two groups. Analysis of covariance was used to adjust for WHR. Pearson correlations were used in the correlation analysis. A $P$ value $\leq 0.05$ was considered as statistically significant.

\section{Results}

\section{Baseline patient characteristics}

The clinical, hormonal, and metabolic features of the PCOS women and controls are shown in Table 1. The age and BMI were well matched in the two groups. The mean WHR was significantly higher in the PCOS group $(P<0.001)$. As expected, the androgen levels were significantly higher among the women with PCOS ( $P<0.001$ for T, fT, A, and DHEAS). The groups did not differ, with regard to total cholesterol, TG, and Lp-a values. Although HDL-C levels were within the normal range for both groups, women with PCOS had significantly lower values $(P<0.001)$. The PCOS group had significantly higher fasting insulin, $\mathrm{AUC}_{\text {glucose, }} \mathrm{AUC}_{\text {insulin' }}$ and HOMA IR values than the control group $(P<0.001, P<$ $0.01, P<0.001$, and $P<0.001$, respectively). FG:I was significantly lower in the PCOS group $(P<0.01)$.

\section{Coagulation and fibrinolysis}

GFC was found to be significantly lower in the PCOS group, compared with the control group $(P<0.001)$. All the other coagulation and fibrinolysis parameters (including PT; aPTT; TT; ATIII; factors II, V, VII, and X; fibrinogen; PLG act; $\mathrm{AP}$; and D-dimer) were comparable between the two groups (Table 2). After adjustment for WHR, GFC remained significantly lower in the PCOS group (analysis of covariance, $P<$ 0.05); and WHR-adjusted mean differences for all the other

TABLE 1. The clinical, hormonal, and metabolic features of the women with PCOS and controls in the study

\begin{tabular}{|c|c|c|c|}
\hline & $\begin{array}{c}\text { PCOS } \\
(\mathrm{n}=59)\end{array}$ & $\begin{array}{l}\text { Controls } \\
(\mathrm{n}=23)\end{array}$ & $P$ \\
\hline Age (yr) & $22.9 \pm 4.4$ & $24.8 \pm 4.2$ & NS \\
\hline BMI $\left(\mathrm{kg} / \mathrm{m}^{2}\right)$ & $23.0 \pm 2.4$ & $22.1 \pm 2.2$ & NS \\
\hline WHR & $0.76 \pm 0.02$ & $0.71 \pm 0.02$ & $<0.001$ \\
\hline $\mathrm{T}(\mathrm{nM})$ & $6.0 \pm 1.8$ & $2.3 \pm 0.8$ & $<0.001$ \\
\hline $\mathrm{fT}(\mathrm{pM})$ & $11.4 \pm 3.8$ & $4.8 \pm 1.0$ & $<0.001$ \\
\hline $\begin{array}{l}\text { Androstenedione } \\
\text { (nmol/liter) }\end{array}$ & $9.4 \pm 3.1$ & $5.9 \pm 1.7$ & $<0.001$ \\
\hline DHEAS $(\mu \mathrm{M})$ & $8.62 \pm 2.58$ & $5.33 \pm 1.50$ & $<0.001$ \\
\hline Total cholesterol (mM) & $4.66 \pm 0.80$ & $4.38 \pm 0.65$ & NS \\
\hline $\begin{array}{l}\text { Triglycerides } \\
\text { (mmol/liter) }\end{array}$ & $1.05 \pm 0.58$ & $0.81 \pm 0.37$ & NS \\
\hline HDL-C (mmol/liter) & $1.37 \pm 0.26$ & $1.74 \pm 0.34$ & $<0.001$ \\
\hline $\mathrm{Lp}-\mathrm{a}(\mathrm{mg} / \mathrm{dl})$ & $21 \pm 12$ & $12 \pm 7$ & NS \\
\hline $\begin{array}{l}\text { Fasting glucose } \\
(\mathrm{mmol} / \mathrm{liter})\end{array}$ & $5.1 \pm 0.7$ & $4.8 \pm 0.4$ & NS \\
\hline $\begin{array}{l}\text { Fasting insulin } \\
(\mathrm{pmol} / \mathrm{liter})\end{array}$ & $70.2 \pm 29.4$ & $44.4 \pm 13.8$ & $<0.001$ \\
\hline $\begin{array}{l}\mathrm{AUC}_{\text {glucose }} \\
\text { ( } \mathrm{mmol} / \text { /iter} / \mathrm{min})\end{array}$ & $793 \pm 137$ & $665 \pm 97$ & $<0.01$ \\
\hline $\begin{array}{l}\mathrm{AUC}_{\text {insulin }} \\
\quad(\mathrm{pmol} / \text { liter/min })\end{array}$ & $36900 \pm 15732$ & $19656 \pm 6036$ & $<0.001$ \\
\hline HOMA IR & $2.57 \pm 1.16$ & $1.56 \pm 0.52$ & $<0.001$ \\
\hline FG:I (mg/10 $\left.{ }^{-4} \mathrm{U}\right)$ & $9 \pm 4.4$ & $13.4 \pm 4.8$ & $<0.01$ \\
\hline
\end{tabular}

Data are presented as the mean \pm SD. NS, Not significant.
TABLE 2. Coagulation and fibrinolysis parameters of the women with PCOS and controls in the study

\begin{tabular}{lccc}
\hline & $\begin{array}{c}\text { PCOS } \\
(\mathrm{n}=59)\end{array}$ & $\begin{array}{c}\text { Control } \\
(\mathrm{n}=23)\end{array}$ & $P$ \\
\hline PT (sec) & $13.4 \pm 1.1$ & $13.5 \pm 1$ & $\mathrm{NS}$ \\
aPTT (sec) & $32.9 \pm 4.3$ & $33.5 \pm 2.7$ & $\mathrm{NS}$ \\
TT (sec) & $16.5 \pm 1.6$ & $17.3 \pm 3.7$ & $\mathrm{NS}$ \\
AT III $(\%)$ & $106 \pm 13$ & $103 \pm 16$ & $\mathrm{NS}$ \\
Factor II $(\%)$ & $88 \pm 18$ & $93 \pm 16$ & $\mathrm{NS}$ \\
Factor V (\%) & $81 \pm 23$ & $93 \pm 28$ & $\mathrm{NS}$ \\
Factor VII $(\%)$ & $85 \pm 23$ & $96 \pm 26$ & $\mathrm{NS}$ \\
Factor X $(\%)$ & $85 \pm 19$ & $86 \pm 15$ & $\mathrm{NS}$ \\
Fibrinogen $(\mathrm{mg} / \mathrm{dl})$ & $319 \pm 64$ & $305 \pm 36$ & $\mathrm{NS}$ \\
PLG act $(\%)$ & $91 \pm 16$ & $89 \pm 15$ & $\mathrm{NS}$ \\
AP $(\%)$ & $106 \pm 23$ & $102 \pm 18$ & $\mathrm{NS}$ \\
D-dimer $(\mathrm{ng} / \mathrm{ml})$ & $0.27 \pm 0.16$ & $0.26 \pm 0.11$ & $\mathrm{NS}$ \\
GFC $(\mu \mathrm{g} / \mathrm{ml})$ & $2.49 \pm 1.6$ & $5.95 \pm 2.43$ & $<0.001$ \\
\hline
\end{tabular}

Data are presented as the mean $\pm \mathrm{SD}$.

coagulation and fibrinolysis parameters were comparable between the two groups.

\section{Correlation analyses}

GFC was correlated with $\mathrm{T}$ and $\mathrm{fT}$ negatively and with HDL-C positively $(\mathrm{r}=-0.486, P=0.001 ; \mathrm{r}=-0.371, P=$ 0.014 ; and $r=0.327, P=0.04$, respectively). There was no correlation between GFC and any of the IR parameters, including fasting insulin, $\mathrm{AUC}_{\text {insulin, }}$ HOMA-IR, and FG:I values.

\section{Discussion}

In this study, GFC in women with PCOS was assessed for the first time. GFC, as reflected by the amount of D-dimer after aprotinin, was lower in the PCOS group than in the healthy control group. All the other coagulation and fibrinolysis parameters were comparable between the two groups. Although GFC was correlated with androgens negatively and HDL-C positively, there was no correlation between GFC and any of the IR parameters.

Women with PCOS have risk factors for premature CVD (4). The relative risk of myocardial infarction in women with PCOS was reported to be 7.4 times that of age-matched controls (8). A 4-fold increase in the relative risk of myocardial infarction in postmenopausal women with a history of ovarian wedge resection for PCOS at age 40-49 yr and an 11-fold increase at age 50-60 yr were found, in a retrospective study (16). Two studies from the same cohort reported findings in women with PCOS that were diagnosed in the United Kingdom before $1979(5,6)$. Women were followed historically for an average of $30 \mathrm{yr}$; standardized mortality ratios were calculated to compare the death rates with general population rates. All-cause and cardiovascular mortality in the cohort were similar to that of women in the general population, and a history of coronary heart disease was not significantly more common. Several cardiovascular risk factors (including diabetes, hypertension, lipid abnormalities, increased WHR, and a history of CVD) were more prevalent among women with PCOS $(5,6)$.

The impact of hypercoagulability on CVD is well-established (29-31). The suppression of fibrinolysis precedes CVD. High plasma concentrations of PAI-1, factor VII, fi- 
brinogen, $\mathrm{vWF}$, and tPA are associated with the development of myocardial infarction $(19,32)$.

Previous studies have reported conflicting data about coagulation and fibrinolysis in PCOS. Dahlgren et al. (16) studied 28 PCOS women (43-62 yr old) and 56 controls. There was no difference in PAI-1, vWF, factor VII activity, and fasting insulin levels. Fibrinogen levels were significantly lower, and the prevalence of hypertension and diabetes were significantly higher in the PCOS group in this study. Sampson et al. (14) evaluated 24 nonobese women with PCO on ultrasound and menstrual disturbance, 26 matched controls with a normal menstrual cycle and PCO on ultrasound, and 10 matched controls with a normal menstrual cycle and normal ovarian ultrasound. They found that median fasting plasma insulin was significantly higher in the PCOS group than the other groups. Although the PCOS group had higher PAI-1 values, the difference between the PCOS and control groups was not significant. There was a significant relation between fasting plasma insulin and PAI-1 activity. Atiomo et al. (15) studied 11 women with PCOS and 12 controls. They found that women with PCOS had significantly higher PAI-1 activity and fibrinogen levels, compared with controls. There was no significant difference in plasminogen and $\alpha 2 \mathrm{AP}$ levels. The mean values of BMI in PCOS and control groups were 31 and $25.9 \mathrm{~kg} / \mathrm{m}^{2}$, and the difference was significant. In another study by the same group (17), 41 women with PCOS and 25 healthy controls were evaluated. Although the PCOS group had significantly higher fasting plasma insulin and lower HDL-C/total cholesterol ratio, compared with controls, there was no significant difference in PAI-1 activity, plasminogen, $\alpha 2 \mathrm{AP}$, and fibrinogen concentrations.

In our study, PT; aPTT; TT; ATIII; factors II, V, VII, and X; fibrinogen; PLG act; AP; and D-dimer were comparable between the PCOS and control groups. Only GFC was found to be lower among the women with PCOS. Decreased GFC reflects impaired fibrinolysis, which could be attributable to alterations of any of the proteins involved in fibrinolysis. The biological process of fibrinolysis is a great dynamic puzzle. A wide variety of stimulatory and inhibitory molecules involved in hemostasis comprise the puzzle and affect some part of the overall fibrinolytic response, either in a negative or a positive way. Many previous studies about fibrinolysis, in any pathological event (including PCOS), dissected the whole picture by focusing on a cluster of profibrinolytic and/or antifibrinolytic molecules. GFC, is a novel assay reflecting the net fibrinolytic response, resulted in the dynamic interactions of numerous stimulatory and inhibitory molecules. In our present study; molecules affecting fibrinolysis (such as plasminogen, AP, or fibrinogen) were similar between PCOS patients and controls. However, GFC was decreased as an indicator of a state of hypofibrinolysis. Those findings implicate that GFC can be important in indicating prothrombotic states.

In our study, glucose intolerance or obesity were not confounding factors to hypofibrinolysis in PCOS. GFC was correlated with androgens negatively; and HDL-C, positively. The Thrombocheck Study (23), performed in a large population, made known that GFC is lower in males than in females. In our study, the lower GFC values in women with PCOS vs. healthy controls and the negative correlation be- tween GFC vs. testosterone and fT values indicates a relationship between hypofibrinolysis and hyperandrogenemia; however, it is not rational to assign GFC levels to a simple androgen effect from this cross-sectional study. Contrary to the expectation, GFC was not related to IR. This lack of association indicates that hypofibrinolysis is an independent risk factor of thrombosis. GFC is a complex and multifactorial end-parameter of the dynamic process of fibrinolysis. A wide variety of (patho)biological factors may affect its level; nevertheless, reduced GFC could implicate hypofibrinolysis/ fibrinolytic dysfunction in a given clinical setting. The Thrombocheck Study (23) has shown that GFC was correlated with several major cardiovascular risk factors in a healthy population. There was an inverse correlation between GFC and BMI, glycemia, TG; and a direct correlation between GFC and HDL-C. GFC was significantly lowered in hypercholesterolemia, and this diminution was enhanced in the presence of an associated hypertriglyceridemia. GFC was even further reduced in noninsulin-dependent diabetes mellitus. The low GFC in hyperlipidemia and diabetes has indicated that GFC was sensitive to the deficient fibrinolysis associated with these conditions, which carry a significant cardiovascular risk (23). Patients with mitral valve prolapse and decreased GFC have been shown to have transient ischemic attacks as a thrombotic microvascular complication (26).

Contributions of several physiological influences on GFC have been assessed. There is no effect of smoking on GFC in a previous investigation in which oral contraceptive users had higher GFC values (22). Glycemia and sport practice also contributed significantly to GFC (33). Because GFC is a novel method, it is clear that further studies will shed light on the exact relationships between many (patho)physiologic influences and GFC.

The fibrinolytic system is the principal effector of clot removal. Insufficient activation of fibrinolysis, as reflected by decreased GFC, represents a thrombotic tendency in patients with PCOS. Impairment of the fibrinolytic system interferes with the hemostatic balance and can lead to thrombosis by inhibiting clot resolution. Regarding the findings of hypofibrinolysis in the pathobiology of coronary thrombosis (31, 34), decreased global fibrinolysis in PCOS must be considered as an important finding in explaining CVD in this syndrome. Ovarian steroidogenesis systems, together with intraovarian and circulating IGF systems should be searched to elucidate hypofibrinolysis of PCOS. The IGF system is involved in the pathogenesis of PCOS, and IGF-1 can cause hypofibrinolysis $(35,36)$. Similar interactions must be investigated for other molecules that present in the cyst fluids of PCOS.

Our results suggest that women with PCOS have impaired fibrinolysis, as reflected by the decreased GFC. This impairment is not related to IR and can contribute to the risk of CVD in PCOS. The issue is not just academical. Elucidation of the modifiable mechanisms and significance of hypofibrinolysis in PCOS can represent an opportunity for preventive therapy of cardiovascular risks associated with PCOS (37). Further investigations are needed to elucidate whether this impairment is modifiable and to shed light on the importance of hypofibrinolysis in PCOS and CVD. 


\section{Acknowledgments}

Received November 13, 2001. Accepted April 17, 2002.

Address all correspondence and requests for reprints to: Bülent $\mathrm{O}$. Yildiz, Emeklisubayevleri 2. C 23 C Blok, No: 15/3 06580 Yucetepe, Ankara, Turkey. E-mail: yildizbo@yahoo.com.

\section{References}

1. Dunaif A 1997 Insulin resistance and the polycystic ovary syndrome: mechanism and implications for pathogenesis. Endocr Rev 18:774-800

2. Knochenhauer ES, Key TJ, Kahsar-Miller M, Waggoner W, Boots LR, Azziz R 1998 Prevalence of the polycystic ovary syndrome in unselected black and white women of the southeastern United States: a prospective study. J Clin Endocrinol Metab 83:3078-3082

3. Diamanti-Kandarakis E, Kouli CR, Bergiele AT, Filandra FA, Tsianateli TC, Spina GG, Zapanti ED, Bartzis MI 1999 A survey of the polycystic ovary syndrome in the Greek island of Lesbos: hormonal and metabolic profile. J Clin Endocrinol Metab 84:4006-4011

4. Wild RA 2002 Polycystic ovary syndrome: a risk for coronary artery disease? Am J Obstet Gynecol 186:35-43

5. Pierpoint T, McKeigue PM, Isaacs AJ, Wild SH, Jacobs HS 1998 Mortality of women with polycystic ovary syndrome at long-term follow-up. J Clin Epidemiol 51:581-586

6. Wild S, Pierpoint T, McKeigue P, Jacobs H 2000 Cardiovascular disease in women with polycystic ovary syndrome at long-term follow-up: a retrospective cohort study. Clin Endocrinol (Oxf) 52:595-600

7. Conway GS, Agrawal R, Betteridge DJ, Jacobs HS 1992 Risk factors for coronary artery disease in lean and obese women with the polycystic ovary syndrome. Clin Endocrinol (Oxf) 37:119-125

8. Dahlgren E, Janson PO, Johansson S, Lapidus L, Oden A 1992 Polycystic ovary syndrome and risk for myocardial infarction. Evaluated from a risk factor model based on a prospective population study of women. Acta Obstet Gynecol Scand 71:599-604

9. Dahlgren E, Johansson S, Lindstedt G, Knutsson F, Oden A, Janson PO, Mattson LA, Crona N, Lundberg PA 1992 Women with polycystic ovary syndrome wedge resected in 1956 to 1965: a long-term follow-up focusing on natural history and circulating hormones. Fertil Steril 57:505-513

10. Guzick DS 1996 Cardiovascular risk in women with polycystic ovarian syndrome. Semin Reprod Endocrinol 14:45-49

11. Mather KJ, Kwan F, Corenblum B 2000 Hyperinsulinemia in polycystic ovary syndrome correlates with increased cardiovascular risk independent of obesity. Fertil Steril 73:150-156

12. Talbott E, Guzick D, Clerici A, Berga S, Detra K, Weimer K, Kuller L 1995 Coronary heart disease risk factors in women with polycystic ovary syndrome. Arterioscler Thromb Vasc Biol 15:821-826

13. Talbott EO, Guzick DS, Sutton-Tyrrell K, McHugh Pemu KP, Zborowski JV, Remsberg KE, Kuller LH 2000 Evidence for association between polycystic ovary syndrome and premature carotid atherosclerosis in middle-aged women. Arterioscler Thromb Vasc Biol 20:2414-2421

14. Sampson M, Kong C, Patel A, Unwin R, Jacobs HS 1996 Ambulatory blood pressure profiles and plasminogen activator inhibitor (PAI-1) activity in lean women with and without the polycystic ovary syndrome. Clin Endocrinol (Oxf) 45:623-629

15. Atiomo WU, Bates SA, Condon JE, Shaw S, West JH, Prentice AG 1998 The plasminogen activator system in women with polycystic ovary syndrome. Fertil Steril 69:236-241

16. Dahlgren E, Janson PO, Johansson S, Lapidus L, Lindstedt G, Tengborn L 1994 Hemostatic and metabolic variables in women with polycystic ovary syndrome. Fertil Steril 61:455-460

17. Atiomo WU, Fox R, Condon JE, Shaw S, Friend J, Prentice AG, Wilkin TJ 2000 Raised plasminogen activator inhibitor-1 (PAI-1) is not an independent risk factor in the polycystic ovary syndrome (PCOS). Clin Endocrinol (Oxf) 52: 487-492

18. Atiomo WU, Condon J, Adekanmi O, Friend J, Wilkin TJ, Prentice AG 2000 Are women with polycystic ovary syndrome resistant to activated protein C? Fertil Steril 74:1229-1232

19. Thompson SG, Kienast J, Pyke SD, Haverkate F, van de Loo JC $1995 \mathrm{He}-$ mostatic factors and the risk of myocardial infarction or sudden death in patients with angina pectoris. European Concerted Action on Thrombosis and Disabilities Angina Pectoris Study Group. N Engl J Med 332:635-641

20. Amiral J 1996 The global fibrinolytic capacity. Fibrinolysis 10:95

21. Scarabin PY, Alhenc-Gelas M, Plu-Bureau G, Taisne P, Agher R, Aiach M 1997 Effects of oral and transdermal estrogen/progesterone regimens on blood coagulation and fibrinolysis in postmenopausal women. A randomized controlled trial. Arterioscler Thromb Vasc Biol 17:3071-3078

22. Scarabin PY, Vissac AM, Kirzin JM, Bourgeat P, Amiral J, Agher R, Guize L 1999 Elevated plasma fibrinogen and increased fibrin turnover among healthy women who both smoke and use low-dose oral contraceptives-a preliminary report. Thromb Haemost 82:1112-1116

23. Amiral J, Malmejac A, Gin H, Pannel R, Vissac A-M, Seigneur M, Scarabin PY, Boisseau M, Guize L, Gurewich V 1999 Evaluation of the fibrinolytic potential on plasma: physiological and pathological variations, and associations with cardiovascular disease risk factors. Fibrinolysis Proteol 13:1-10

24. Yurdakok M, Yigit S, Korkmaz A, Kirazli S, Aygun C 2001 Global fibrinolytic capacity in healthy newborn infants. Turk J Pediatr 43:177-179

25. Ozatli D, Sayinalp N, Buyukasik Y, Karakus S, Haznedaroglu IC, Kirazli S, Ozcebe O, Dundar SV 2002 Unchanged global fibrinolytic capacity despite increased factor VIIa activity in Behcet's disease: evidence of a prethrombotic state. Rheumatol Int 21:137-140

26. Atalar E, Acil T, Aytemir K, Haznedaroglu IC, Ozer N, Kilic H, Kuru G, Aksoyek S, Ovunc K, Kes S, Kirazli S, Ozmen F 2002 Diminished global fibrinolytic capacity in patients with mitral valve prolapse is associated with transient ischemic attacks. Clin Appl Thromb Hemost 8:41-44

27. Expert Committee on the Diagnosis and Classification of Diabetes Mellitus 1997 Report of the Expert Committee on the Diagnosis and Classification of Diabetes Mellitus. Diabetes Care 20:1183-1197

28. Matthews DR, Hosker JP, Rudenski AS, Naylor BA, Treacher DF, Turner RC 1985 Homeostasis model assessment: insulin resistance and beta-cell function from fasting plasma glucose and insulin concentrations in man. Diabetologia 28:412-419

29. Ambrose JA, Weinrauch M 1996 Thrombosis in ischemic heart disease. Arch Intern Med 156:1382-1394

30. DeLoughery TG 1999 Coagulation abnormalities and cardiovascular disease. Curr Opin Lipidol 10:443-448

31. Juhan-Vague I, Collen D 1992 On the role of coagulation and fibrinolysis in atherosclerosis. Ann Epidemiol 2:427-438

32. Meade TW, Mellows S, Brozovic M, Miller GJ, Chakrabarti RR, North WR, Haines AP, Stirling Y, Imeson JD, Thompson SG 1986 Haemostatic function and ischaemic heart disease: principal results of the Northwick Park Heart Study. Lancet 2:533-537

33. Amiral J 1997 The global fibrinolytic capacity. Blood Coagul Fibrinolysis 8:20

34. Fareed J, Hoppensteadt DA, Jeske WP, Ahmad S, Bick RL 1999 Acquired defects of fibrinolysis associated with thrombosis. Semin Thromb Hemost 25:367-374

35. Thierry van Dessel HJ, Lee PD, Faessen G, Fauser BC, Giudice LC 1999 Elevated serum levels of free insulin-like growth factor I in polycystic ovary syndrome. J Clin Endocrinol Metab 84:3030-3035

36. Padayatty SJ, Orme S, Zenobi PD, Stickland MH, Belchetz PE, Grant PJ 1993 The effects of insulin-like growth factor- 1 on plasminogen activator inhibitor-1 synthesis and secretion: results from in vitro and in vivo studies. Thromb Haemost 70:1009-1013

37. Lobo RA, Carmina E 2000 The importance of diagnosing the polycystic ovary syndrome. Ann Intern Med 132:989-993 